Pacific Journal of Mathematics

GENERALIZED ROTATIONAL HYPERSURFACES OF
CONSTANT MEAN CURVATURE IN THE EUCLIDEAN SPACES.

WU-YI HSIANG AND HSUEH-LING HUYNH 


\title{
GENERALIZED ROTATIONAL HYPERSURFACES OF CONSTANT MEAN CURVATURE IN THE EUCLIDEAN SPACES, II
}

\author{
WU-YI HSIANG AND HSUEH-LING HUYNH
}

\begin{abstract}
Among various basic local differential geometric invariants of a given hypersurface in the euclidean $(n+1)$-space, $M^{n} \subset E^{n+1}$, the mean curvature, i.e. the trace of the second fundamental form, is certainly one of the simplest numerical invariants with important geometric meaning, namely, the first variation of "area". Therefore, complete hypersurfaces of constant mean curvatures in $E^{n+1}$ naturally constitute a nice family of simple global geometric objects that certainly deserve special attention. Especially, those closed ones can be considered as natural generalizations of "soap bubbles" and the problem of such generalized soap bubbles in the euclidean spaces has been attracting the attention of differential geometers since Euler and Monge.

This paper is the second part of a systematic study of hypersurfaces of constant mean curvatures in $E^{n+1}$ which are of generalized rotational types, succeeding a previous paper with the same title.
\end{abstract}

Historically, the study of rotational surface of constant mean curvature in $E^{3}$ can be traced back to the work of Delaunay in 1841 [8], in which he discovered a beautiful way of constructing the generating curves of such rotational surfaces, namely, the trace of a focus by rolling a given conic section on the axis.

In the paper preceding this one [I], it was proposed to generalize the study of rotational surfaces in the setting of invariant hypersurfaces of a given isometric transformation group $\left(G, E^{n+1}\right)$ with two-dimensional orbit space, namely, hypersurfaces of generalized rotational types. Such isometric transformation groups, $\left(G, E^{n+1}\right)$, of cohomogeneity 2 were classified in [13] and they can be naturally divided into five types according to the geometric shape of their orbit spaces, which are linear cones of angle $\pi / g$, $g=1,2,3,4$ and 6 respectively. The case $g=1$ consists of the usual rotational transformation groups, $\left(O(n), E^{n+1}\right)$, and it was proved in [16] that the generating curves of $O(n)$-invariant hypersurfaces of constant mean curvatures can again be obtained by rolling construction, a straightforward generalization of the above mentioned theorem of Delaunay. The next case of $g=2$ consists of those transformation groups $(O(p) \times$ $\left.O(q), E^{p+q}\right), p, q \geq 2$, and this is exactly the case studied in the paper preceding this one [I] . One of the main results of $[\mathbf{I}]$ is the existence of 
infinitely many non-congruent $O(p) \times O(q)$-invariant immersions of $S^{p+q-1} \rightarrow E^{p+q}$ with constant mean curvature 1 for each given pair of $p, q \geq 2$. The purpose of this paper is to extend the results of [I] not only to all the remaining cases of cohomogeneity two orthogonal transformation groups with $g=3,4$ and 6 , but also to all cases of rank 2 isoparametric foliations. Roughly speaking, isoparametric foliations of euclidean spaces are a kind of "geometric abstraction" of the orbital foliations of the isotropy representations of symmetric spaces. In particular, the family of rank 2 isoparametric foliations of euclidean spaces constitutes a geometric generalization of the family of orbital foliations of cohomogeneity 2 orthogonal transformation groups classified in [13], and hence, foliated hypersurfaces of such a given foliation are a kind of further generalization of those generalized rotational types proposed in [I] .

Let $\mathscr{F}$ be a given rank 2 isoparametric foliation of $E^{n+2}, x_{0}$ an arbitrarily chosen generic point, and $\mathscr{F}\left(x_{0}\right)$ the leaf passing through $x_{0}$. Then the normal plane of $\mathscr{F}\left(x_{0}\right)$ at $x_{0}, E_{x_{0}}^{2}$, intersects perpendicularly with every leaf of $\mathscr{F}$ and has an induced transformation group of Coxeter type [20] (cf. $\$ 2 \mathrm{~A}$ ). Let $C_{0}$ be a Weyl chamber of the above associated Coxeter group, $\left(W, E_{x_{0}}^{2}\right)$, and $M^{n+1}$ is a foliated hypersurface in $E^{n+2}$. Then $C_{0}$ is a wedge region of angle $\pi / g, g=1,2,3,4$ or 6 , which intersects every leaf of $\mathscr{F}$ exactly once, and hence, a foliated hypersurface $M^{n+1}$ is uniquely determined by its generating curve, $M^{n+1} \cap C_{0}$. In $\S 2$ we shall show that the generating curves of foliated hypersurfaces of constant mean curvature $h$ are characterized by a specific ODE (cf. $\$ 2 C$ ). We state the main results of this paper in terms of the geometric behavior of the global solution curves of the specific $\operatorname{ODE}(*, \mathscr{F})$ of $\S 2 \mathrm{C}$.

Theorem A. Let $\gamma=\{\gamma(s)=(r(s), \theta(s)) ;-\infty<s<+\infty\}$ be $a$ global solution curve of $(*, \mathscr{F})$ parametrized by its arc length $s$. Then there exist two constants $d_{1}(h, \mathscr{F})$ and $d_{2}(h, \mathscr{F})$ such that

$$
\begin{aligned}
& \lim _{s \rightarrow-\infty} r(s) \cdot \sin \theta(s)=d_{1}(h, \mathscr{F}), \\
& \lim _{s \rightarrow+\infty} r(s) \sin \left(\frac{\pi}{g}-\theta(s)\right)=d_{2}(h, \mathscr{F}),
\end{aligned}
$$

namely, every global solution curve has the following two straight lines

$$
\begin{aligned}
& l_{1}=\left\{(r, \theta) ; r \sin \theta=d_{1}(h, \mathscr{F})\right\}, \\
& l_{2}=\left\{(r, \theta) ; r \sin \left(\frac{\pi}{g}-\theta\right)=d_{2}(h, \mathscr{F})\right\}
\end{aligned}
$$

as its asymptotic lines. 
THEOREM B. Every global solution curve, $\gamma$, can hit the boundary line $\theta=0$ (resp. $\theta=\pi / g$ ) at most once. There is a well-defined winding number of $\gamma, n(\gamma)$, such that the total change of direction of $\gamma$ is given as follows

$$
\sigma_{\gamma}(+\infty)-\sigma_{\gamma}(-\infty)=(2 n(\gamma)-1) \pi+\frac{\pi}{g}
$$

where $\sigma_{\gamma}(s)$ denotes the directional angle of $\gamma$ at $\gamma(s)$ and $\sigma_{\gamma}( \pm \infty)$ are the limiting values of $\sigma_{\gamma}(s)$ as $s \rightarrow \pm \infty$.

REMARK. By our definition, each instance of the solution curve $\gamma$ hitting $\partial C_{0}$ will contribute 1 to the winding number $n(\gamma)$. Therefore, it is not difficult to show that $n(\gamma) \geq$ the number of boundary points on $\gamma$.

THEOREM C. (i) To each integer $k \geq 0$, there exists a global solution curve $\gamma$ with $n(\gamma)=k$ and not hitting $\partial C_{0}$. (ii) To each $k \geq 1$, there exists $a$ global solution curve $\gamma$ with $n(\gamma)=k$ and hitting $\partial C_{0}$ exactly once at the line $\theta=0$ (resp. $\theta=\pi / g$ ). (iii) To each $k \geq 2$, there exists a global solution curve $\gamma$ with $n(\gamma)=k$ and hitting $\partial C_{0}$ twice.

THEOREM D. There exist infinitely many non-congruent $\mathscr{F}$-foliated immersions of $S^{n+1} \rightarrow E^{n+2}$ with constant mean curvature 1 , for each rank two isoparametric foliation, $\mathscr{F}$, of $E^{n+2}$ with $g=2,3,4$ or 6 .

REMARKS. (i) In the case $g=2$, the above four theorems constitute the main results proved in [I]. In this paper, we shall provide a unified proof of the above results which cover all cases of rank two isoparametric foliations with $g=2,3,4$ or 6 .

(ii) In the special case of $g=2$, there exist two auxiliary functions (denoted by $I$ and $J$ in [I]) which are automatically monotonic along any given solution curve. These two auxiliary functions together with their monotonicity play a crucial role throughout the whole proof of [I]. However, in the cases $g=3,4$ or 6 , the corresponding auxiliary functions are no longer monotonic! It is exactly the lack of such monotonicity for the cases $g=3,4$ and 6 that makes it necessary to seek a different approach other than that of [I], especially in the proof of Theorem A.

(iii) The above four theorems describe some of the major geometric features of the "generating curves" of foliated hypersurfaces of constant mean curvatures. Conceptually, they constitute an extension of the classical theorem of Delaunay to considerably greater generality, which also happens to include many varieties of new examples of "soap bubbles". 
(iv) The techniques developed in this paper can also be applied to the study of soap bubbles in symmetric spaces, cf. [12].

\section{Isoparametric foliations of the Euclidean spaces and foliated} hypersurfaces of constant mean curvature.

\section{A. Isoparametric Foliations of the Euclidean Spaces.}

An isoparametric hypersurface in a space of constant curvature (i.e., a euclidean, spherical or hyperbolic Riemannian manifold) is, by definition, a level surface of an isoparametric function, namely, a function $f$ : $M^{n+1}(c) \rightarrow \mathbf{R}$ satisfying $\Delta f$ and $|\nabla f| \equiv 0(\bmod f)$. Geometrically, the level surfaces of such an isoparametric function constitutes a "parallel" foliation of hypersurfaces of constant mean curvatures. We shall call such a foliation a codimension one isoparametric foliation of $M^{n+1}(c)$. Such nice geometric structures were first studied by B. Segre and É. Cartan. In a series of papers $[3,4,5,6]$ of É. Cartan, he was particularly fascinated by the profound depth of the spherical case and its mysterious connection with the Lie group theory. This subject was somehow forgotten until it was revived by a sequence of recent papers by Nomizu [19], Münzner [18], Ozeki-Takeuchi [21], Ferus-Karcher-Münzner [9]. Higher codimensional generalization of isoparametric foliations was proposed in a recent paper of Terng [22], namely, a submanifold $N^{n}$ in $M^{n+k}(c)$ is called isoparametric if its normal bundle is flat and the principal curvatures in the directions of any parallel normal vector field are constant; an isoparametric foliation of $M^{n+k}(c)$ is, by definition, a parallel foliation by isoparametric submanifolds and their focal varieties. We refer to [22] for a general theory as well as some basic theorems on isoparametric foliations in $E^{n+k}$.

A typical example of isoparametric foliation (which, in fact, motivates its definition, cf. [22]) is the orbital foliation of the adjoint action of a compact connected Lie group $G$ on its Lie algebra $g$. It follows from the maximal torus theorem of $\dot{E}$. Cartan that the above orbital foliation has the following basic geometric properties:

(i) the principal orbit type is $G / T$,

(ii) the Lie algebra of a given maximal torus, $T$, i.e. a Cartan subalgebra $\mathfrak{h}$, intersects every $G$-orbit perpendicularly,

(iii) the Weyl group $W=N(T) / T$ acts on $\mathfrak{h}$ as a group generated by reflections and $\mathfrak{g} / G \cong \mathfrak{h} / W$.

The above result was generalized by É. Cartan himself to the setting of the isotropy representation of a symmetric space $M=G / K$, namely,

(i) a normal plane, $\mathfrak{h}$, to an arbitrary principal $K$-orbit in $\mathfrak{p}$ automatically intersects all $K$-orbits perpendicularly 
(ii) there is an induced Coxeter group $(W, \mathfrak{h})$ such that $\mathfrak{p} / K \cong \mathfrak{h} / W$. Let $M^{n}$ be a given isoparametric submanifold in $E^{n+k}$ and $E_{x}^{k}$ be the normal plane of $M^{n}$ at a point $x \in M^{n}$. Choose an arbitrary base point $x_{0}$ on $M^{n}$. Then $E_{x_{0}}^{k}$ intersects $M^{n}$ perpendicularly at a finite number of points and, moreover, there is a unique parallel translation $\Pi_{x_{1}, x_{2}}: E_{x_{0}}^{k}=$ $E_{x_{1}}^{k} \rightarrow E_{x_{2}}^{k}=E_{x_{0}}^{k}$ for each given pair of points $x_{1}, x_{2} \in E_{x_{0}}^{k} \cap M^{n}$, thus generating an induced transformation group $\left(W, E_{x_{0}}^{k}\right)$. It was proved in [22] that $\left(W, E_{x_{0}}^{k}\right)$ is automatically a group generated by reflections, called the associated Coxeter group of $M^{n} \subset E^{n+k}$. It follows from a theorem of Chevalley [7] that the ring of $W$-invariant polynomials of $E_{x_{0}}^{k} \simeq \mathbf{R}^{k}$ is a free algebra generated by $k$ homogeneous generators, say $\left\{u_{i} ; 1 \leq i \leq k\right\}$. It was proved in [22] that each $u_{i}$ extends uniquely to a homogeneous polynomial on $E^{n+k} \simeq \mathbf{R}^{n+k}$ which is constant on $M^{n}$ and moreover,

$$
\mathbf{u}=\left(u_{1}, \ldots, u_{k}\right): E^{n+k} \rightarrow \mathbf{R}^{k}
$$

defines an "isoparametric map" whose level surfaces form an isoparametric foliation of $E^{n+k}$. As one can see from the above brief account of isoparametric foliations, it is exactly a kind of "geometric abstraction" of the orbital foliations of these isotropy representations of symmetric spaces.

B. Foliated Submanifolds of a Given Isoparametric Foliation.

For a given codimension $k$ isoparametric foliation on $E^{n+k}$, let $\left(W, \mathbf{R}^{k}\right)$ be its associated Coxeter group and $p: E^{n+k} \rightarrow \mathbf{R}^{k} / W$ be the associated projection map. By definition, the above, map, $p$, is a Riemannian submersion of a particularly simple kind, namely, in the terminology of O'Neill [20], the submersion tensor $A$ is identically zero and the second fundamental form tensor $T$ is constant along each generic fiber. A foliated submanifold of $E^{n+k}$ is, by definition, a submanifold which consists of a suitable subcollection of leaves. Hence, a foliated submanifold, $M^{n+d}$, intersects with the normal section, $\mathbf{R}^{k}$, transversally and is uniquely determined by the above intersection, $M^{n+d} \cap \mathbf{R}^{k}$.

The tangent bundle of each isoparametric submanifold $M^{n}$ in $E^{n+k}$ has a splitting as the orthogonal direct sum of the subspaces of equiprincipal-directions:

$$
T M^{n}=\bigoplus \sum\left\{E_{j} ; \operatorname{dim} E_{j}=m_{j}, 1 \leq j \leq g\right\}
$$

The integral submanifold of $E_{j}$ passing $x_{0} \in M^{n} \cap \mathbf{R}^{k}$ is a round sphere of dimension $m_{j}$ which intersects $\mathbf{R}^{k}$ at the antipodal point of $x_{0}$, say $x_{j}$, and moreover, $\left\{\Pi_{x_{0} x_{j}} ; 1 \leq j \leq g\right\}$ are exactly those generating reflections 
of $W$. We shall call $m_{j}$ the marked multiplicity of the $j$ th reflection hyperplane, $H_{j}$, and call the above structure a Coxeter group with marked multiplicities. Observe that the projection map $p: E^{n+k} \rightarrow \mathbf{R}^{k} / W$ is a Riemannian submersion with $A \equiv 0$ and $T$ completely determined by the above Coxeter group with marked multiplicities. Therefore, it forms a complete set of invariants for the local geometry of the given isoparametric foliation. In particular, it is quite straightforward to write down the second fundamental form of a foliated submanifold, $M^{n+d} \subset E^{n+k}$, in terms of the second fundamental form of its image, $\bar{M}^{d} \subset \mathbf{R}^{k} / W$, and the following set of distance functions, namely,

$$
\left\{\rho_{j}(x)=\begin{array}{l}
\text { the distance between } x \text { and the } \\
j \text { th hyperplane } H_{j}, 1 \leq j \leq g
\end{array}\right\}
$$

where $x \in \bar{M}^{d} \subset \mathbf{R}^{k} / W$ and $\mathbf{R}^{k} / W$ is identified with a chosen Weyl chamber of $\left(W, \mathbf{R}^{k}\right)$.

C. Rank Two Isoparametric Foliations and Foliated Hypersurfaces of Constant Mean Curvatures.

In this paper, we shall mainly exploit those rank two isoparametric foliations on euclidean spaces to construct families of examples of constant mean curvature hypersurfaces. For a given rank two isoparametric foliation on $E^{n+2}$, its associated Coxeter group is a dihedral group, $\left(W ; \mathbf{R}^{2}\right)$, whose fundamental domain, $\mathbf{R}^{2} / W$, is a wedge with its angle equal to $\pi / g$, $g=1,2,3,4$ or 6 [cf. 13]. Since the cases $g=1$ and 2 have already been treated in [16] and [I] respectively, we shall only discuss here the remaining cases of $g=3,4$ and 6 as follows:

(i) If $g=3$, then it was proved by É. Cartan $[4,5]$ that there are exactly four cases with uniform marked multiplicities $1,2,4$ and 8 respectively. They are the orbital foliations of $\left(\mathrm{SO}(3), \mathbf{R}^{5}\right),\left(\mathrm{SU}(3), \mathbf{R}^{8}\right)$, $\left(\mathrm{Sp}(3), \mathbf{R}^{14}\right)$ and $\left(F_{4}, \mathbf{R}^{26}\right)$ respectively.

(ii) If $g=6$, then it was proved by Abresch [1] that they must also be with uniform marked multiplicities 1 or 2 .

(iii) The case $g=4$ is certainly the most involved and also the most interesting one. There exist a large family of non-orbital examples obtained by means of orthogonal representations of Clifford algebras [9] and the exact extent of possibilities of marked multiplicities is still not yet completely determined. We quote here the following theorem of Abresch [1]:

THEOREM 2.1 [Abresch]. Let $m_{-} \leq m_{+}$be the marked multiplicities of the two generating reflection lines of a given rank two isoparametric foliation 
with $g=4$. Then they satisfy one of the following three conditions:

(4A): $\quad m_{+}+m_{-}+1$ is divisible by $2^{K}:=\min \left\{2^{\sigma} \mid 2^{\sigma}>m_{-}\right\}$,

(4B1): $m_{-}$is a power of 2 and $2 m_{-}$divides $m_{+}+1$,

(4B2): $\quad m_{-}$is a power of 2 and $3 m_{-}=2 \cdot\left(m_{+}+1\right)$.

Let $M^{n+1}$ be a foliated hypersurface of constant mean curvature in $E^{n+2}, C_{0} \cong \mathbf{R}^{2} / W$ be a chosen Weyl chamber of its associated Coxeter group $\left(W, \mathbf{R}^{2}\right)$ and $\gamma=M^{n+1} \cap C_{0}$ be the generating curve of $M^{n+1}$. Then one has the following characterizing ODE of $\gamma$, namely,

LEMMA 2.1. In terms of the polar coordinates $(r, \theta), 0 \leq r<\infty$, $0 \leq \theta \leq \pi / g$, the above generating curve, $\gamma=C_{0} \cap M^{n+1}$, of a foliated hypersurface of constant mean curvature $h$ is characterized by the following $O D E(*, \mathscr{F})$ :

(i) the case $g=3: k=1,2,4,8$

$(*)_{3} \quad \frac{d \sigma}{d s}=(3 k+1) h$

$$
+\frac{k}{r}\left\{\frac{\cos \sigma}{\sin \theta}-\frac{\sin (\sigma+\pi / 6)}{\cos (\theta+\pi / 6)}-\frac{\sin (\sigma-\pi / 6)}{\cos (\theta-\pi / 6)}\right\}
$$

(ii) the case $g=6: k=1,2$

$(*)_{6} \frac{d \sigma}{d s}=(6 k+1) h$

$$
\begin{array}{r}
+\frac{k}{r}\left\{\frac{\cos \sigma}{\sin \theta}-\frac{\sin \sigma}{\cos \theta}-\frac{\sin (\sigma+\pi / 6)}{\cos (\theta+\pi / 6)}-\frac{\sin (\sigma-\pi / 6)}{\cos (\theta-\pi / 6)}\right. \\
\left.-\frac{\sin (\sigma+\pi / 3)}{\cos (\theta+\pi / 3)}-\frac{\sin (\sigma-\pi / 3)}{\cos (\theta-\pi / 3)}\right\}
\end{array}
$$

(iii) the case $g=4:\{k, l\}=\left\{m_{+}, m_{-}\right\}$, (cf. Theorem 1.1$)$

$(*)_{4}$

$$
\begin{aligned}
\frac{d \sigma}{d s}= & (2 k+2 l+1) h+\frac{k}{r}\left\{\frac{\cos \sigma}{\sin \theta}-\frac{\sin \sigma}{\cos \theta}\right\} \\
& -\frac{l}{r}\left\{\frac{\sin (\sigma+\pi / 4)}{\cos (\theta+\pi / 4)}+\frac{\sin (\sigma-\pi / 4)}{\cos (\theta-\pi / 4)}\right\},
\end{aligned}
$$

where $s$ is the arclength parameter of $\gamma$ and $\sigma$ is the angle between the positive $x$-direction and the tangent direction of $\gamma$. 
Proof. Let $v(r, \theta)$ be the $n$-dimensional volume of the isoparametric submanifold passing through the point $(r, \theta)$. Then it is not difficult to see that

$$
v(r, \theta)=c_{0} \cdot \prod_{i=1}^{g} \rho_{l}(r, \theta)^{m_{t}},
$$

where $c_{0}=$ a suitable constant, $\rho_{i}(r, \theta)$ is the distance between $(r, \theta)$ and the $i$ th reflection line, and $m_{i}$ is its marked multiplicity. Moreover, straightforward computation will show that

$$
(n+1) h=\frac{d \sigma}{d s}-\frac{d}{d n} \ln v(r, \theta)
$$

and hence the characterizing $\operatorname{ODE}(*)_{g}$ for each of the specific cases of $g=3,4$ and 6 .

\section{Some analytical lemmas and the proof of Theorems A and B.}

A. The Analysis of the $O D E(*, \mathscr{F})$ in the Neighborhood of $\partial C_{0}$.

At a boundary point of $C_{0}$, say $(r, 0)$ or $(r, \pi / g)$, the $\operatorname{ODE}(*, \mathscr{F})$ becomes singular because the denominator of one term tends to zero. However, one has the following lemma of existence and uniqueness on solution curves of $(*, \mathscr{F})$ passing through a boundary point:

LeMma 3.1. To each point $P=(r, 0)$ (resp. $Q=(r, \pi / g)$ ), $r>0$, there exists a unique solution curve of $(*, \mathscr{F})$ passing through $P$ (resp. $Q$ ) which forms a perpendicular cusp point at $P$ (resp. $Q$ ) and depends on $r$ analytically.

We refer to Proposition 5 of [I] and Proposition 1 of [11] for a proof of the above Lemma 3.1.

From now on, we shall always assume, for simplicity of notation, that $h=1$. We shall denote the unique solution curve passing through $(b, 0)$ (resp. $(b, \pi / g)$ ) by $\gamma_{(b, 0)}$ (resp. $\gamma_{(b, \pi / g)}$ ) and moreover, use $\gamma_{(b, 0)}^{+}$(resp. $\left.\gamma_{(b, 0)}\right)$ to denote the segment of $\gamma_{(b, 0)}$ which consists of points after (resp. before) the cusp point $(b, 0)$. For a given $b>0$, it follows from Lemma 3.1 that there exists a sufficiently small $\delta(b)>0$ and two coordinate systems $\{(u, y)\}$ and $\{(v, y)\}$, of the $\delta(b)$-collar neighborhood of the segment $\{(r, 0) ; b \leq r<\infty\}$ such that

$$
0 \leq y=r \sin \theta \leq \delta(b)
$$

and $\gamma_{(r, 0)}^{-}\left(\right.$resp. $\left.\gamma_{(r, 0)}^{+}\right)$is exactly given by $u=r$ (resp. $\left.v=r\right)$ for all $b \leq r<\infty$. 
In such a $\delta(b)$-collar region, the value of $r \sin \theta$ is very small and hence the curvature, $d \sigma / d s$, of a solution curve inside this region is dominated by the term $k \cos \sigma / r \sin \theta$. Therefore, unless $\gamma$ is, in fact, the curve $u=r_{0}$, a solution curve $\gamma$ will make a rapid turn back as it approaches the boundary $\theta=0$, as indicated in the following Figure 1.

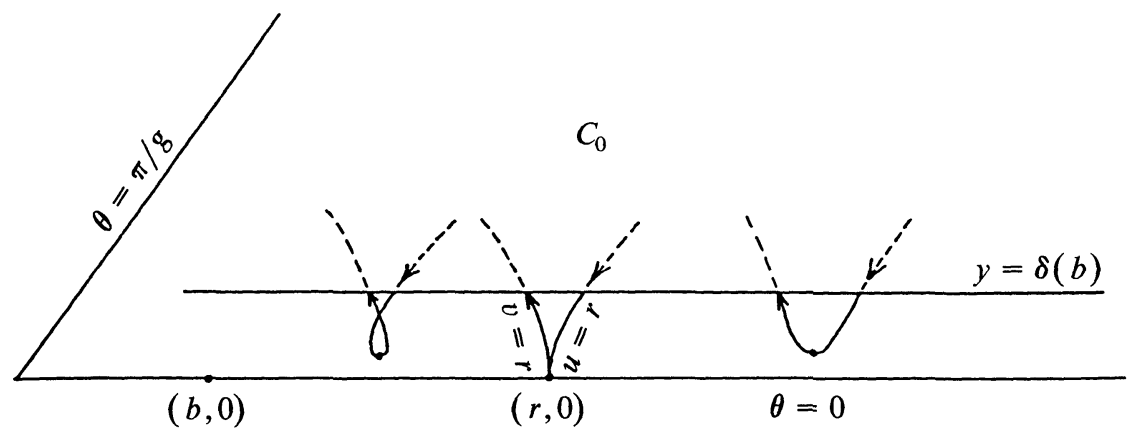

Figure 1

LEMMA 3.2. Let $\gamma_{t}, 0<t \leq 1$, be a continuous family of solution curves of $(*, \mathscr{F}), u(t)$ (resp. $v(t))$ be the u-coordinate (resp. $v$-coordinate) of the entrance (resp. exit) point of $\gamma_{t}$ and $(\hat{u}(t), y(t))$ be the $(u, y)$-coordinate of the unique $y$-minimum point of $\gamma_{t}$. If $y(t)>0$ for all $0<t \leq 1$ and as $t \rightarrow 0$

$$
\lim y(t)=0, \quad \lim \hat{u}(t)=r_{0}
$$

then the directions of $\gamma_{t}$ at the $y$-minimum point, $0<t \leq 1$, must be the same and $\lim u(t)=\lim v(t)=r_{0}$ as $t \rightarrow 0$.

Proof. Since $y(t)$ is assumed to be positive for all $0<t \leq 1$, it follows directly from the continuity of $\gamma_{t}$ that the direction of $\gamma_{t}$ at the $y$-minimum point must be the same.

Let $t_{n}$ be an arbitrary sequence with $\lim t_{n}=0$. Without loss of generality, one may assume that, as $n \rightarrow \infty$,

$\lim u\left(t_{n}\right), \lim v\left(t_{n}\right), \quad \lim \sigma_{n}$ and $\lim \sigma_{n}^{\prime}$ all exist

where $\sigma_{n}$ and $\sigma_{n}^{\prime}$ are respectively the entrance and exit direction of $\gamma_{t_{n}}$. Then it follows from the assumptions $\lim y(t)=0, \lim \hat{u}(t)=r_{0}$, and Lemma 3.1 that

$$
\lim u\left(t_{n}\right)=\lim v\left(t_{n}\right)=r_{0}
$$


and $\lim \sigma_{n}$ (resp. $\left.\lim \sigma_{n}^{\prime}\right)$ must be equal to the entrance (resp. exit) direction of $\gamma_{\left(r_{0}, 0\right)}$. For otherwise, those $\gamma_{t_{n}}$ with large $n$ should be a $U$-shaped curve with the bottom part coasting very closely along the singular boundary $\theta=0$.

Such a solution curve is impossible because the dominating large value of the term $k \cos \sigma / r \sin \theta$ will make any solution curve sharply turn away from the line $\theta=0$.

\section{B. The Analysis of Two Auxiliary Functions.}

In analyzing the geometric behavior of global solution curves of $(*, \mathscr{F})$ for the special case of $g=2$ in $[\mathbf{I}]$, there are two auxiliary functions, denoted by $I$ and $J$, which play the key role throughout the discussion of $[\mathbf{I}]$. One of the crucial properties of these two auxiliary functions of $[\mathbf{I}]$ is their monotonicity along any given solution curve (cf. Proposition 4 of [I]). In this subsection, we shall again introduce the following two auxiliary functions and study their properties along certain specific segments of solution curves.

Definition. For $g=3,4$ and 6 , set $n=3 k, 2 k+2 l$ and $6 k$ respectively; and let $k^{\prime}=k$ if $g=3$ or $6, k^{\prime}=l$ if $g=4$. Define

$$
\begin{gathered}
I=\left[r \cos \left(\theta+\frac{\pi}{2}-\frac{\pi}{g}\right)\right]^{k^{\prime}} \sin \left(\sigma+\frac{\pi}{2}-\frac{\pi}{g}\right) \\
-\frac{n+1}{k^{\prime}+1}\left[r \cos \left(\theta+\frac{\pi}{2}-\frac{\pi}{g}\right)\right]^{k^{\prime}+1}, \\
J=[r \sin \theta]^{k} \cos \sigma+\frac{n+1}{k+1}[r \sin \theta]^{k+1}=y^{k} \cos \sigma+\frac{n+1}{k+1} y^{k+1} .^{*}
\end{gathered}
$$

As we shall see, the behavior of $\gamma$ as $s \rightarrow-\infty$ is governed through an analysis of $J$, in the same way that $I$ governs the behavior of $\gamma$ as $s \rightarrow+\infty$. Henceforth we shall only exhibit our lemma for $J$.

Since $\dot{y}=\sin \sigma$, we have

$$
\frac{d J}{d s}=y^{k} \dot{y}\left[(n+1)+\frac{k \cos \sigma}{y}-\dot{\sigma}\right]
$$

where the dot denotes differentiation with respect to $s$. Thus we see that $J$ is a first integral of the equation

$$
\dot{\sigma}=(n+1)+\frac{k \cos \sigma}{y},
$$

\footnotetext{
* In the ensuing discussion, both rectangular coordinates $(x, y)$ and polar coordinates $(r, \theta)$ will be used as convenient.
} 
obtained from the $\operatorname{ODE}(*, \mathscr{F})$ by removing the terms contributed by all but one factor in the volume function (see the proof of Lemma 2.1). These equations were thoroughly studied in [16]. In particular, the asymptotic line as $s \rightarrow-\infty$ stated in Theorem $\mathrm{A}$ is a solution of the above equation with $\dot{\sigma}=0$.

LEMMA 3.3. Along a given solution curve of $O D E(*, \mathscr{F})$ :

(i)

$$
\frac{d J}{d s}=\left\{\begin{array}{l}
k y^{k} \cdot \frac{\sin \sigma[3 \cos \theta \sin \sigma+\sin \theta \cos \sigma]}{r\left(\frac{1}{2}+\cos 2 \theta\right)} \quad \text { for } g=3 ; \\
y^{k} \cdot \frac{\sin \sigma[((k+l) \cos 2 \theta+l) \sin \sigma+l \sin 2 \theta \cos \sigma]}{r \cos 2 \theta \cos \theta} \quad \text { for } g=4 ; \\
k y^{k} \cdot \frac{\sin \sigma\left[3 \frac{\cos 4 \theta}{2}+\frac{\cos 2 \theta}{2}+\frac{1}{4} \sin \sigma+\sin 4 \theta+\frac{\sin 2 \theta}{2} \cos \sigma\right]}{r \cos \theta\left(\cos 2 \theta+\frac{1}{2}\right)\left(\cos 2 \theta-\frac{1}{2}\right)} \quad \text { for } g=6 .
\end{array}\right.
$$

(ii) The ranges of non-monotonicity of $J$ are given as follows: $(d J / d s)<$ 0 if and only if $\pi-\bar{\alpha}<\sigma<\pi$ or $\alpha<\sigma<0$, where

$$
\tan \bar{\alpha}(\theta)= \begin{cases}1 / 3 \tan \theta & \text { for } g=3 ; \\ \frac{\sin 2}{[(k+l) \cos 2 \theta+l]} & \text { for } g=4 ; \\ \frac{\left[\sin 4 \theta+\frac{\sin 2 \theta}{2}\right]}{3\left[\frac{\cos 4 \theta}{2}+\frac{\cos 2 \theta}{2}+\frac{1}{4}\right]} & \text { for } g=6 .\end{cases}
$$

Proof. (i) For $g=3$, compare the expression for $d J / d s$ in Remark (ii) with the $\operatorname{ODE}(*, \mathscr{F})$; we have

$$
\frac{d J}{d s}=\frac{k y^{k} \dot{y}}{r}\left\{\frac{\sin (\sigma+\pi / 6)}{\cos (\theta+\pi / 6)}+\frac{\sin (\sigma-\pi / 6)}{\cos (\theta-\pi / 6)}\right\} \text {. }
$$

The assertion follows by direct computation, noting that $\dot{y}=\sin \sigma$. For $g=4$ or 6 , the proof is similar. (ii) follows easily from (i).

REMARK. For $g=3,4$ and 6 ,

$$
J \geq-\left[\frac{k}{n+1}\right]^{k} \frac{1}{k+1} .
$$


The lower bounds are attained by the straight lines $y=k /(n+1)$, which will be shown to be the asymptotic lines of Theorem A.

Let us now consider a segment of a fixed solution curve $\gamma$ of the ODE $(*, \mathscr{F})$ which contains a local maximum for $y_{\gamma}$ at $s=s_{1}$ followed by a local minimum for $y_{\gamma}$ at $s=s_{0}$. We will allow $\gamma\left(s_{0}\right)$ to be a boundary point, viz. $y_{\gamma}\left(s_{0}\right)=0$. Referring to Figure 2, we define certain functions of $y$ for $y_{\gamma}\left(s_{0}\right) \leq y \leq y_{\gamma}\left(s_{1}\right)$. Set $s_{0}>s(y)>s_{1}>s^{\prime}(y)$ so that $y_{1} \geq$ $y(s)=y\left(s^{\prime}\right) \geq y_{0}$. Let $\alpha(y)=\sigma(s)-\pi$ and $\alpha^{\prime}(y)=\pi-\sigma\left(s^{\prime}\right)$. Set $J(y)$ $=J(s), J^{\prime}(y)=J\left(s^{\prime}\right)$. We define $s^{\prime}, \alpha^{\prime}, J^{\prime}$ only when $s^{\prime}$ is in one-to-one correspondence with $y$, which means that they are defined where $0 \leq \alpha^{\prime}$ $\leq \pi / 2$.

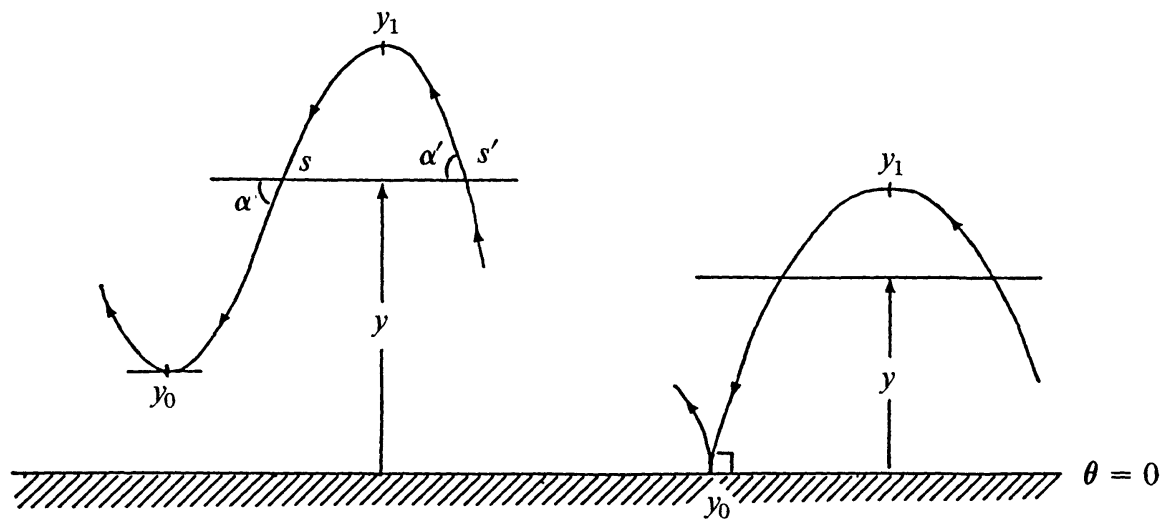

FIGURE 2

We have come to the important cancellation lemma that helps to overcome the non-monotonicity of $J$.

LEMMA 3.4. For $y<y_{1}$ and when $s^{\prime}$ is defined,

$$
J(y)>J^{\prime}(y), \quad \alpha(y)>\alpha^{\prime}(y) .
$$

Proof. First observe that $J=(n+1) /(k+1) y^{k+1}-\cos \alpha \cdot y^{k}$, and a similar relation holds for $J^{\prime}, \alpha^{\prime}$. Hence the two inequalities in the lemma are equivalent. Now

$$
J(y)-J^{\prime}(y)=\int_{y}^{y_{1}}\left[-\frac{d J}{d y}\right]_{s}-\left[-\frac{d J}{d y}\right]_{s^{\prime}} d y .
$$

It suffices to show that the integrand is positive. 
Recall Lemma 3.3(i). For $g=3$,

$$
\begin{aligned}
& {\left[-\frac{d J}{d y}\right]_{s}=k y^{k} \frac{[3 \cos \theta \sin \alpha+\sin \theta \cos \alpha]}{r\left(\frac{1}{2}+\cos 2 \theta\right)},} \\
& {\left[-\frac{d J}{d y}\right]_{s^{\prime}}=k y^{k} \frac{\left[-3 \cos \theta^{\prime} \sin \alpha^{\prime}+\sin \theta^{\prime} \cos \alpha^{\prime}\right]}{r^{\prime}\left(\frac{1}{2}+\cos 2 \theta^{\prime}\right)} .}
\end{aligned}
$$

It is clear that $r<r^{\prime}$ and $\theta>\theta^{\prime}$, so that the denominator of $[-d J / d y]_{s}$ is greater than the denominator of $[-d J / d y]_{s^{\prime}}>0$. We need only show that

$$
[3 \cos \theta \sin \alpha+\sin \theta \cos \alpha]-\left[-3 \cos \theta^{\prime} \sin \alpha^{\prime}+\sin \theta^{\prime} \cos \alpha^{\prime}\right]>0 .
$$

But

the last quantity $>3 \cos \theta \sin \alpha+\cos \alpha \sin \theta-\sin \theta^{\prime}$

$$
\begin{aligned}
& >\{3 \cos \theta \sin \alpha+\cos \alpha \sin \theta\}-\sin \theta, \quad \text { since } \theta>\theta^{\prime}, \\
& >\min \{3 \cos \theta-\sin \theta, \sin \theta-\sin \theta\} \geq 0 \text { for } 0 \leq \theta \leq \frac{\pi}{3},
\end{aligned}
$$

since the minimum of the sum of the positive terms occur either at $\alpha=0$ or $\alpha=\pi / 2$.

For $g=4$, the same argument reduces the assertion to showing that

$$
\begin{aligned}
& {[((k+l) \cos 2 \theta+l) \sin 6+l \sin 2 \theta \cos \alpha]} \\
& \quad-\left[-\left((k+l) \cos 2 \theta^{\prime}+l\right) \sin \alpha^{\prime}+l \sin 2 \theta^{\prime} \cos \alpha^{\prime}\right]>0 .
\end{aligned}
$$

The last quantity $>\min \{(k+l) \cos 2 \theta+l-l \sin 2 \theta, l \sin 2 \theta-l \sin 2 \theta\}$ $\geq 0$ for $0 \leq \theta \leq \pi / 4$.

For $g=6$, we need to show that

$$
\begin{gathered}
{\left[3\left(\frac{\cos 4 \theta}{2}+\frac{\cos 2 \theta}{2}+\frac{1}{4}\right) \sin \alpha+\left(\sin 4 \theta+\frac{\sin 2 \theta}{2}\right) \cos \alpha\right]} \\
-\left[-3\left(\frac{\cos 4 \theta^{\prime}}{2}+\frac{\cos 2 \theta^{\prime}}{2}+\frac{1}{4}\right) \sin \alpha^{\prime}\right. \\
\left.+\left(\sin 4 \theta^{\prime}+\frac{\sin 2 \theta^{\prime}}{2}\right) \cos \alpha^{\prime}\right]>0 .
\end{gathered}
$$

The last quantity $>\min \left\{3\left(\frac{\cos 4 \theta}{2}+\frac{\cos 2 \theta}{2}+\frac{1}{4}\right)-\left(\sin 4 \theta+\frac{\sin 2 \theta}{2}\right)\right.$,

$$
\left.\left(\sin 4 \theta+\frac{\sin 2 \theta}{2}\right)-\left(\sin 4 \theta+\frac{\sin 2 \theta}{2}\right)\right\}
$$

$\geq 0$ for $0 \leq \theta \leq 25^{\circ}$. 
Although $25^{\circ}$ falls short of the full range for $\theta$, this estimate suffices for our purpose, as it is impossible for a local maximum of $y$ to occur with the specified orientation, and with $\theta>25^{\circ}$. In fact, the present case compares favorably with the minimal equation $(h=0)$ for the same group of transformations, where such behavior is established in [10] (cf. Proposition 1 , especially paragraph (b)).

As a simple but important consequence of the fact $\alpha(y)>\alpha^{\prime}(y)$, we have the following

COROLlaRY 3.4. There is a local minimum for $y_{\gamma}$ at $s_{2}$ with $s_{2}<s_{1}$ and $y_{\gamma}\left(s_{2}\right)>y_{\gamma}\left(s_{0}\right)$.

Proof. Since $s_{1}$ is a local maximum of $y_{\gamma}$, clearly there is an $s_{2}<s_{1}$ such that either $y_{\gamma}\left(s_{2}\right)=0$ or $s_{2}$ is a local minimum of $y_{\gamma}$, and furthermore that all points on $\gamma$ between $s_{1}$ and $s_{2}$ are neither local $y$-minima nor boundary points.

Let us assume $y_{\gamma}\left(s_{2}\right)=0$, or $y_{\gamma}\left(s_{2}\right)$ is a local minimum but with $y_{\gamma}\left(s_{2}\right)<y_{\gamma}\left(s_{0}\right)$. In either case, we can find an $s^{\prime}$ such that $s_{0}>s_{1}>s>s_{2}$ and $y_{\gamma}\left(s_{0}\right)=y_{0}=y_{\gamma}\left(s^{\prime}\right)$. But then $\alpha\left(y_{0}\right)=0, \alpha\left(y_{0}\right)=\sigma(s)-\pi>0$. This contradicts Lemma 3.4 and proves the corollary.

\section{The Proof of Theorems A and B.}

In order to establish Theorem A, namely, that all global solution curves of the $\operatorname{ODE}(*, \mathscr{F})$ are asymptotic to the line $y=k /(n+1)$ as $s \rightarrow-\infty$ (and the corresponding statement for $s \rightarrow+\infty$ ), let us first record a lemma which is, in essence, covered in [I].

LEMMA 3.5. Suppose $\gamma$ is a solution curve of the $O D E(*, \mathscr{F})$ such that, as $s \rightarrow-\infty, y_{\gamma}$ remains bounded, $x_{\gamma} \rightarrow+\infty$, and $J \leq 0$. Then $\gamma$ is asymptotic to $y=k /(n+1)$ as $s \rightarrow-\infty$.

Proof (sketch). Recall that the ODE $(*, \mathscr{F})$ takes the form

$$
\dot{\sigma}=C+\frac{k \cos \sigma}{y}+\phi(x, y, \sigma)
$$

where $C$ is a constant and $\phi(x, 1, \sigma) \rightarrow 0$ as $x \rightarrow+\infty$ and $y$ bounded.

Now

$$
J=\frac{C}{k+1} y^{k+1}+\cos \sigma \cdot y^{k}, \quad \text { hence } \frac{d J}{d s}=y^{k} \dot{y} \phi(x, y, \sigma) .
$$


If $\gamma$ behaves as in the hypothesis, then for a sufficiently large $s_{0}, s>s_{0}$ implies that $0<y<M$ and $|\phi(x, y, \sigma)|<\delta / M^{k+1}$ for some prescribed $\delta>0$. Whence

$$
\begin{aligned}
\left|J_{\gamma}(s)-J_{\gamma}\left(s_{0}\right)\right| & =\left|\int_{s_{0}}^{s} \phi y^{k} \dot{y} d s\right|=\left|\int_{y\left(s_{0}\right)}^{y(s)} \phi y^{k} d y\right| \\
& <\frac{\delta}{M^{k+1}} M^{k}\left|y(s)-y\left(s_{0}\right)\right| \leq \delta .
\end{aligned}
$$

Thus the curve $\gamma$ is closely approximated by a curve defined by $J=J_{\gamma}\left(s_{0}\right)$ $\leq 0$, which is an $x$-translational invariant, slowly oscillating curve; when $J_{\gamma}\left(s_{0}\right)=-[k /(n+1)]^{k}(k+1)$, i.e. the infimum for $J$, this curve is the line $y=k(n+1)$.

Moreover, when $s$ is very large and negative, $\theta_{\gamma}$ is very small. By Lemma 3.3(ii), the range of $\sigma$ for which $(d J / d s)<0$ appears only for a glimpse.

Let us assume, contrary to the desired conclusion, that $\gamma$ is not asymptotic to the line $y=k /(n+1)$. Then we can remove segments of $\gamma$ including parts where $(d J / d s)<0$, and apply the estimate in the proof of Theorem 1 [I] to obtain a contradiction; for details, we refer the reader to that paper.

In [I], by virtue of the monotonicity of the analogous auxiliary function $J$, one can easily see that $\mid J$ - negative constant $\mid \leq \delta$ eventually $(s \rightarrow-\infty)$ for all solution curves. Here we shall first prove this for a certain class of solution curves, and then extend the conclusion of the theorem to all curves by continuity, noticing that asymptoticity is a closed property relative to the deformation of solutions.

LEMMA 3.6. Consider a solution curve $\gamma$ such that for one value $s_{0}$ of $s$, $y_{\gamma}\left(s_{0}\right)$ is a local minimum of $y_{\gamma}$ with $\sigma_{\gamma}\left(s_{0}\right)=\pi$, or $y_{\gamma}\left(s_{0}\right)=0$, and also $J_{\gamma}\left(s_{0}\right) \leq 0 . \gamma$ is asymptotic to $y=k /(n+1)$ as $s \rightarrow-\infty$.

Proof. First we argue that there is a local maximum of $y$ at some $\gamma\left(s_{1}\right)$ with $s_{1}<s_{0}$. Assume the contrary; then as $s \rightarrow-\infty$, either $y$ increases without bound or $\gamma$ approaches a horizontal line asymptotically from below. The latter possibility is dismissed since solution curves with large $x$ and bounded $y$ are oscillatory. For the former possibility, $J \rightarrow$ $+\infty$ as $s_{0}>s \rightarrow-\infty$. However, note that $d J / d s \geq 0$ for $\pi<\sigma<3 \pi / 2$. Also, the situation depicted in Figure 3 is prohibited by the differential equation. Together it means that $J$ would decrease as $s_{0}>s \rightarrow-\infty$, which is a contradiction. 


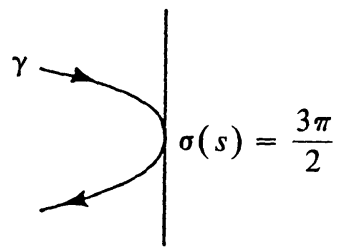

FIGURE 3

We are now placed in the hypothesis of Lemma 3.4. Apply Corollary 3.4 and the above argument repeatedly. We obtain a succession of $y$-local minima $y_{0} \leq y_{2} \leq y_{4} \leq \cdots$ as $s \rightarrow-\infty$; and, in between, a succession of $y$-local maxima $y_{1}, y_{3}, y_{5}, \ldots$ Let $s_{i}$ be the corresponding parameter values to these points. Note also the points $\gamma\left(\bar{s}_{i}\right)$ where $s_{i-1}>\bar{s}_{i}>s_{i}(i$ an odd integer) and $y\left(\bar{s}_{i}\right)=y\left(s_{i+1}\right)$.

By Lemma 3.3(ii), $J\left(s_{i-1}\right)>J\left(\bar{s}_{i}\right)>J\left(s_{i}\right)$. By Lemma 3.4, $J\left(\bar{s}_{i}\right)>$ $J\left(s_{i+1}\right)$. Hence $0 \geq J_{0} \geq J_{2 k+1}$ for all $k$. This implies that as $s \rightarrow-\infty$, $J \leq 0$ and $y$ remains bounded. It is also obvious that $x \rightarrow+\infty$. The required asymptoticity of $\gamma$ now follows from Lemma 3.5.

Observe that $r=1$ is a solution of the $\operatorname{ODE}(*, \mathscr{F})$. It is part of a global solution curve $\Gamma_{2}$ with a point where $y=0$; as an immediate corollary of the above lemma, we have

LEMMA 3.7. The solution curve $\Gamma_{2}$ is asymptotic to the line $r \sin \theta=$ $k /(n+1)$ as $s \rightarrow-\infty$, and to the line $r \sin (\pi / g-\theta)=k^{\prime} /(n+1)$ as $s \rightarrow+\infty$.

A feature new to the cases $g=3,4$ or 6 is the possible appearance of points of inflection on a solution curve, where $\dot{y}=0$ and $\ddot{y}=0$. In the previous case $g=2$ of [I] the last condition would force the solution curve to be the horizontal line. We restrict our attention to those points where $\sigma=\pi$ and $\dot{\sigma}=0$.

LEMMA 3.8. (i) The above mentioned type of point of inflection along any solution curve occurs on the locus defined by

$$
\begin{cases}y=\frac{k}{(3 k+1)}\left\{1-\frac{2 y^{2}}{3 x^{2}-y^{2}}\right\} & \text { for } g=3 \\ y=\frac{k}{(2 k+2 l+1)}-\frac{2 y^{2} l}{(2 k+2 l+1)\left(x^{2}-y^{2}\right)} & \text { for } g=4 \\ y=\frac{k}{(6 k+1)}\left\{1-\frac{2 y}{3 x^{2}-y^{2}}-\frac{6 y^{2}}{x^{2}-3 y^{2}}\right\} & \text { for } g=6\end{cases}
$$


(ii) Given any point on the locus, there exists a unique solution curve having this point as a point of inflection.

(ii) The set of solution curves possessing a point of inflection of the above type is closed.

(iv) $A$ solution curve having a point of inflection of this type is asymptotic to $y=k /(n+1)$ as $s \rightarrow-\infty$.

Proof. (i) The locus is obtained by putting $\sigma=\pi, \dot{\sigma}=0$ into ODE $(*, \mathscr{F})$. (ii) is immediate and (iii) follows from (i) and (ii).

For (iv) let $\gamma\left(s_{0}\right)$ be the point of inflection. It is easy to see that $y_{\gamma}$ is in fact increasing with increasing $s$ near $\gamma\left(s_{0}\right)$, hence there is a $y$-local minimum or a point with $y=0$ at some $\gamma\left(s_{1}\right)$ with $s_{0}>s_{1}$. Then apply Lemma 3.6.

We shall now use crucially the continuity principles established in $\S 3 \mathrm{~A}$. Note that they cover the circumstance under which some of the solution curves may contain boundary points during a continuous change of initial conditions.

LEMMA 3.9. Let $\left\{\gamma_{t}: 0 \leq t \leq 1\right\}$ be a continuous family of global solution curves with $\gamma_{0}=\Gamma_{2}$ (cf. Lemma 3.7). Then $\gamma_{1}$ is asymptotic to $y=k /(n+1)$ as $s \rightarrow-\infty$.

Proof. Let $\Sigma=\left\{t \in(0,1): \gamma_{t}\right.$ is asymptotic to $y=k /(n+1)$ as $s \rightarrow-\infty\} . \Sigma$ is open. Indeed, let $t_{0} \in \Sigma$. Then on $\gamma_{t_{0}}$ we can find a $y$-local minimum $\gamma_{t_{0}}\left(s_{0}\right)$ with $\sigma\left(s_{0}\right)=\pi, \dot{\sigma}\left(s_{0}\right)<0$, and $J\left(s_{0}\right)<0$. These are clearly open conditions, so that such points can be found on curves in a neighborhood of $\gamma_{t_{0}}$. Lemma 3.6 then implies the asymptoticity of these neighboring curves.

Now if $\gamma_{1}$ has a point of inflection, then Lemma 3.8(iv) will give its asymptoticity. Otherwise there is an interval $(1-\varepsilon, 1]$ on which no curve has a point of inflection, by Lemma 3.8(iii) . Furthermore, since $\Sigma$ is open, one may assume without loss of generality that all the curves in $(1-\varepsilon, 1)$ are asymptotic. Let $t \in(1-\varepsilon, 1)$ and $s(t)$ be parameters at which $\gamma_{t}(s(t))$ are $y$-local minima of $\gamma_{t}$ with $J_{\gamma_{t}}(s(t))<0$. If $s(t)$ is unbounded as $t \rightarrow 1$, then we see that $\gamma_{1}$ is governed by Lemma 3.5 and therefore asymptotic. So we assume $s(t) \rightarrow s_{1}<\infty$; three possibilities arise:

(a) $\dot{\sigma}_{\gamma_{t}}(s(t)) \rightarrow 0$. $\gamma_{1}\left(s_{1}\right)$ is a point of inflection of $\gamma_{1}$, contrary to assumption. 
(b) $\dot{\sigma}_{\gamma_{t}}(s(t))$ is bounded and $\nrightarrow 0 . \gamma_{1}\left(s_{1}\right)$ is a $y$-local minimum with $J<0 . \gamma_{1}$ is asymptotic by Lemma 3.6.

(c) $\dot{\sigma}_{\gamma_{t}}(s(t))$ is unbounded. $\gamma_{1}\left(s_{1}\right)$ is a boundary point with the correct orientation, and so $\gamma_{1}$ is asymptotic, again by Lemma 3.6.

Now every global solution curve can be linked to $\Gamma_{2}$ through a one-parameter family, and Theorem A is therefore proved.

The first statement of Theorem B, namely that a solution curve cannot contain two points on the same boundary, is a direct consequence of Corollary 3.4. As in [I], we define $\sigma_{\gamma}$ so that it has a jump of $+\pi$ at a boundary point (cusp). By Theorem A, $\lim _{s \rightarrow+\infty} \sigma_{\gamma}(s)=2 n \pi+(\pi / g)$, whilst $\lim _{s \rightarrow-\infty}=\pi$.

DEFINITION. The integer $n$ associated with a solution curve is called its winding number.

Theorem B follows.

\section{Evolution of global solution curves and the proof of Theorems C and $\mathrm{D}$.}

A. Examples of Some Solution Curves and Their Winding Numbers.

EXAMPLE 4.1. Let $\Gamma_{0}$ be the unique global solution curve with $\theta(0)=$ $\pi / 6, \pi / 12$, and $\mu$ for $g=3,6,4$ respectively, where

$$
\frac{\cot \mu-\tan \mu}{\cot (\mu-\pi / 4)-\tan (\mu-\pi / 4)}=-\frac{l}{k}
$$

$\sigma(0)=\theta(0)+\pi / 2 ; r(0)=\delta$. For sufficiently small $\delta$, there is a small $s<0$ such that $\Gamma_{0}(s)$ is a $y$-local minimum with $\sigma=\pi, J<0$. Therefore no loops are formed for $s<0$. Similar behavior is observed relative to the boundary $\theta=\pi / g$. Hence $n\left(\Gamma_{0}\right)=0$.

EXAmple 4.2. As $\delta \rightarrow 0$ in the last example, the solution curve tends to $\gamma_{0}$ with $r(0)=0$ and $n\left(\gamma_{0}\right)=1$. Let $\gamma$ be a curve with a point on the boundary $\boldsymbol{\theta}=0$. Deforming $\gamma$ through a one-parameter family of curves with cusps towards $\gamma_{0}$, we obtain a curve $\Gamma_{1}$ with a cusp on $\theta=0$ and $n\left(\Gamma_{1}\right)=1$. Similarly we get a $\Gamma_{1}^{\prime}$ with a cusp on $\theta=\pi / g$ and $n\left(\Gamma_{1}^{\prime}\right)=1$.

EXAMPLE 4.3. The curve $\Gamma_{2}$ of Lemma 3.7 has exactly two cusps and $n\left(\Gamma_{2}\right)=2$. The segment between the cusps represents an embedded hypersphere of constant mean curvature. By the theorem of Alexandrov [2], $\Gamma_{2}$ is the unique curve with this property. 
LEMMA 4.4. For every integer $N \geq 0$, there exists a global solution curve $\gamma$ with $n(\gamma) \geq N$.

Proof. We give a proof for $g=3$. The other cases are similar. For $r>9$ and $\pi / 2 \leq \theta \leq \pi / 4$,

$$
\begin{aligned}
|\dot{\sigma}-(3 k+1)| & <\frac{k}{r}\left\{\left|\frac{\cos \sigma}{\theta}\right|+\left|\frac{\sin (\sigma+\pi / 6)}{\cos (\theta+\pi / 6)}\right|+\left|\frac{\sin (\sigma-\pi / 6)}{\cos (\theta-\pi / 6)}\right|\right\} \\
& <\frac{k}{r}\left\{\frac{1}{\sin 15^{\circ}}+\frac{1}{\cos 75^{\circ}}+\frac{1}{\cos 15^{\circ}}\right\} \leq k .
\end{aligned}
$$

Consider a solution curve $\gamma$ with $r(0)=R$ and $\theta(0)=\pi / 6$. Choose $R$ so large that $R>9$ and $(2 k+1) / 2 R \geq(2 N-1) \pi+\pi / 3$.

By Theorem A, there exist $s_{1}<0$ such that $\theta\left(s_{1}\right)=\pi / 12$, and $s_{2}>0$ such that $\theta\left(s_{2}\right)=\pi / 4$. Then

$$
\begin{aligned}
{[\Delta \sigma]_{s_{1}}^{s_{2}} } & =\int_{s_{1}}^{s_{2}} \dot{\sigma} d s \geq \int_{s_{1}}^{s_{2}}(2 k+1) d s \geq(2 k+1) 2 R \sin \frac{\pi}{12} \\
& \geq \frac{(2 k+1)}{2} R \geq(2 N-1) \pi+\frac{\pi}{3} .
\end{aligned}
$$

Since the situation of Figure 3 is untenable on any part of the curve $\gamma$, it cannot " unwind" beyond $s=s_{1}$ and $s=s_{2}$. Hence $n(\gamma) \geq N$.

\section{B. Deformation of Global Solution Curves.}

We shall now obtain all the curves stated in Theorem $\mathrm{C}$ by deformation of known examples, following the method of $\$ 3[\mathbf{I}]$, which we summarize below. It is important to know that the winding number cannot be changed except by a transition through boundary points. The behavior of neighboring curves near a cusp is portrayed in Figure 4.

(1) We start with a curve with a large winding number $N$ guaranteed by Lemma 4.4. It may have no cusps, one cusp, or two cusps.

(2) If it has no cusps, then by deforming it through a one-parameter family towards $\Gamma_{0}$, we obtain as an intermediate curve a $\gamma$ with $n(\gamma)=N$ and $\gamma$ contains one or two cusps.

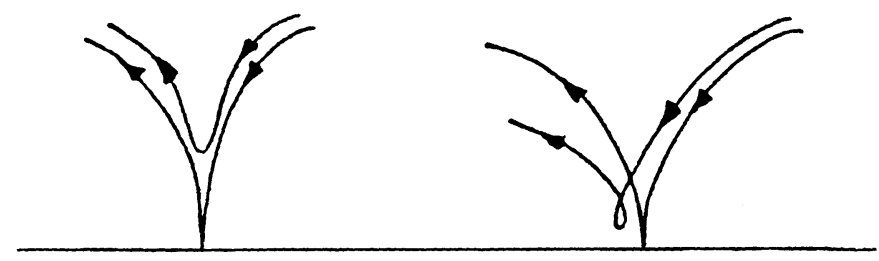


(3) If $\gamma$ has one cusp, by deforming it through a one-parameter family of curves, each having one cusp, towards $\Gamma_{1}$ or $\Gamma_{1}^{\prime}$, we obtain a $\gamma^{\prime}$ with $n\left(\gamma^{\prime}\right)=N$ and two cusps.

(4) Deform $\gamma^{\prime}$ further along curves having one cusp towards $\Gamma_{1}$ or $\Gamma_{1}^{\prime}$, we obtain $\gamma^{\prime \prime}$ with $n\left(\gamma^{\prime \prime}\right)=N-1$ and one cusp.

(5) We may obtain curves with two cusps and a smaller winding number by repeating (3).

(6) Let $\gamma$ be a curve with one cusp, then by a small deformation near the cusp, we get a curve $\gamma^{\prime}$ with no cusp and $n\left(\gamma^{\prime}\right)=n(\gamma)-1$.

These deformations, together with the examples 4.1, 4.2, 4.3 and 4.4, prove Theorem $\mathrm{C}$. The segment of a solution curve between two cusps generates an immersion of $S^{n+1}$ into $E^{n+2}$ with constant mean curvature 1. Examples with unequal winding numbers are clearly non-congruent. This proves Theorem $\mathrm{D}$.

For the topological types of the hypersurfaces generated by other types of curves, we refer the reader to [I].

\section{REFERENCES}

[1] U. Abresch, Isoparametric hypersurfaces with four or six distinct principal curvatures, Math. Ann., 264 (1983), 283-302.

[2] A. D. Alexandrov, A characteristic property of spheres, Ann. Mat. Pura Appl., (4) 58 (1962), 303-315.

[3] É. Cartan, Familles de surfaces isoparamétrique dans les espaces à courbure constante, Ann. Math., 17 (1938), 177-191.

[4] _ Sur des familles remarquables d'hypersurfaces isoparamétriques dans les espaces sphériques, Math. Z., 45 (1939), 335-367.

[5] - Sur quelques familles remarquables d'hypersurfaces, C. R. Congrès Math. Liege, (1939), 30-41.

[6] Sur des familles d'hypersurfaces isoparamétriques des espaces sphériques a 5 et 9 dimension, Univ. Nac. Tucuman Revista A, 1 (1940), 5-22.

[7] C. Chevalley, Invariants of finite groups generated by reflections, Amer. J. Math., 77 (1955), 778-782.

[8] C. Delaunay, Sur la surface de rovólution dont la courbure mayenne est constant, J. Math. Pures Appl., (1) 6 (1841), 309-320.

[9] D. Ferus, H. Karchev and H. F. Münzer, Cliffordalgebren und neue isoparametrische Hyperflächen, Math. Z., 177 (1981), 479-502.

[I] W. Y. Hsiang, Generalized rotational hypersurfaces of constant mean curvature in the Euclidean spaces, I, J. Differential Geom., 17 (1982), 337-356.

[10] _ Minimal cones and the spherical Bernstein problem, II, Inventione Math., 74 (1983), 351-369.

[11] W. Y. Hsiang and W. T. Hsiang, On the existence of codimension one minimal spheres in compact symmetric spaces of rank 2, II, J. Differential Geom., 17 (1982), 583-594.

[12] W. Y. Hsiang and H. L. Huynh, On spherical soap bubbles in non-compact symmetric spaces of rank $\leq 2$, to appear. 
[13] W. Y. Hsiang and B. Lawson, Minimal submanifolds of low cohomogeneity, J. Differential Geom., 5 (1971), 1-38.

[14] W. H. Hsiang, Z. H. Teng, and W. C. Yu, New examples of constant mean curvature immersions of $S^{3}$ into $E^{4}$, Proc. Nat. Acad. Sci. USA, 79 (1982), 3931-3932.

[15] New examples of constant mean curvature immersions of $(2 k-1)$-spheres into Euclidean $2 k$-spaces, Annals of Math., 117 (1983), 609-625.

[16] W. Y. Hsiang and W. C. Yu, A generalization of a theorem of Delaunay, J. Differential Geom., 16 (1981), 161-177.

[17] H. Karcher and D. Ferus, Non-rotational minimal spheres and minimizing cones, preprint.

[18] H. F. Münzer, Isoparametrische Hyperflächen Sparen, I, Math. Ann., 251 (1980), 57-71; II, Math. Ann., 256 (1981), 215-232.

[19] K. Nomizu, Elie Cartan's work on isoparametric families of hypersurfaces, Proc. of Symposia in Pure Math., 27 (AMS) (1975), 191-200.

[20] B. O'Neill, The fundamental equations of a submersion, Michigan Math. J., 13 No. 4, (1966), 459-469.

[21] H. Ozeki and M. Takeuchi, On some types of isoparametric hypersurfaces in spheres, I, Tohoku Math. J., 27 (1975), 515-559.

[22] C. L. Terng, Isoparametric submanifolds and their Coxeter groups, preprint.

Received May 19, 1986 and in revised form November 19, 1986.

UNIVERSITY OF CALIFORNIA

BERKELEY, CA 94720 



\section{PACIFIC JOURNAL OF MATHEMATICS EDITORS}

V. S. VARADARAJAN (Managing Editor) University of California Los Angeles, CA 90024 HERBERT ClEMENS University of Utah Salt Lake City, UT 84112

R. FINN

Stanford University

Stanford, CA 94305

\section{HERMANN FLASCHKA} University of Arizona Tucson, AZ 85721

RAMEsh A. GANGolli University of Washington Seattle, WA 98195

VAUghan F. R. JONES

University of California Berkeley, CA 94720

\section{ROBION KIRBY}

University of California

Berkeley, CA 94720

C. C. MOORE

University of California

Berkeley, CA 94720

HAROLD STARK

University of California, San Diego

La Jolla, CA 92093

\section{ASSOCIATE EDITORS}
R. ARENS
E. F. BECKENBACH
B. H. NEUMANN
F. WOLF
K. YOSHIDA (1906-1982)

\section{SUPPORTING INSTITUTIONS}

UNIVERSITY OF ARIZONA

UNIVERSITY OF BRITISH COLUMBIA

CALIFORNIA INSTITUTE OF TECHNOLOGY

UNIVERSITY OF CALIFORNIA

MONTANA STATE UNIVERSITY

UNIVERSITY OF NEVADA, RENO

NEW MEXICO STATE UNIVERSITY

OREGON STATE UNIVERSITY
UNIVERSITY OF OREGON

UNIVERSITY OF SOUTHERN CALIFORNIA

STANFORD UNIVERSITY

UNIVERSITY OF HAWAII

UNIVERSITY OF TOKYO

UNIVERSITY OF UTAH

WASHINGTON STATE UNIVERSITY

UNIVERSITY OF WASHINGTON 


\section{Pacific Journal of Mathematics}

Vol. 130, No. $1 \quad$ September, 1987

K. Adachi, Continuation of bounded holomorphic functions from certain subvarieties to weakly pseudoconvex domains $\ldots \ldots \ldots \ldots \ldots \ldots \ldots$

Erazm Jerzy Behr, Enveloping algebras of Lie superalgebras ........... 9

Dong M. Chung, Scale-invariant measurability in abstract Wiener spaces . . . 27

Peter Gerard Dodds and Bernardus de Pagter, Algebras of unbounded

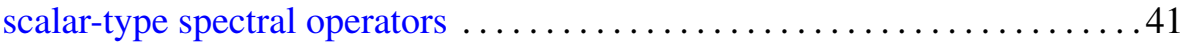

Wu-Yi Hsiang and Hsueh-Ling Huynh, Generalized rotational

hypersurfaces of constant mean curvature in the Euclidean spaces. II . . . .75

Harvey Bayard Keynes and M. Sears, Time changes for $\mathbf{R}^{n}$ flows and suspensions ..................................... 97

Frances Kirwan, Ronnie Lee and Steven Howard Weintraub, Quotients

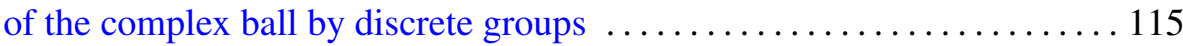

Magnhild Lien, Groups of knots in homology 3-spheres that are not

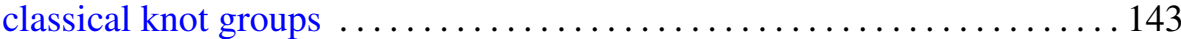

Juan Carlos Migliore, Liaison of a union of skew lines in $\mathbf{P}^{4} \ldots \ldots \ldots \ldots 153$

Jesper M. Møller, Spaces of sections of Eilenberg-Mac Lane fibrations . . . . 171 Daniel Ullman, A generalization of a theorem of Atkinson to noninvariant

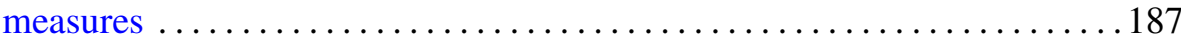

Kohhei Yamaguchi, Operations which detect $\mathscr{P}^{1}$ in odd primary connective

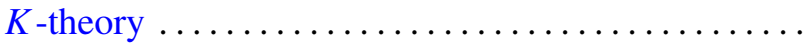

